\title{
Critical role of the adhesion receptor DNAX accessory molecule-1 (DNAM-1) in the development of inflammation-driven dermal fibrosis in a mouse model of systemic sclerosis
}

\author{
Jérôme Avouac, ${ }^{1,2}$ Muriel Elhai, ${ }^{2}$ Michal Tomcik, ${ }^{3,4}$ Barbara Ruiz, ${ }^{2}$ Manuel Friese, ${ }^{5}$ \\ Melanie Piedavent, ${ }^{5}$ Marco Colonna, ${ }^{6}$ Gunter Bernhardt, ${ }^{7}$ André Kahan, ${ }^{1}$ \\ Gilles Chiocchia, ${ }^{2}$ Jörg H W Distler, ${ }^{3}$ Yannick Allanore ${ }^{1,2}$
}

\begin{abstract}
- Additional supplementary files are published online only. To view these files please visit the journal online (http://dx.doi. org/10.1136/annrheumdis2012-201759).

${ }^{1}$ Rheumatology A Department, Paris Descartes University, Sorbonne Paris Cité, Cochin Hospital, Paris, France

${ }^{2}$ Cochin Institute, Paris

Descartes University, INSERM U1016 and CNRS UMR8104,

Paris, France

${ }^{3}$ Department of Internal

Medicine III and Institute for

Clinical Immunology, University

of Erlangen-Nuremberg,

Erlangen, Germany

${ }^{4}$ Department of Rheumatology

of the First Faculty of

Medicine, Institute of

Rheumatology and Connective

Tissue Research Laboratory,

Charles University in Prague,

Czech Republic

${ }^{5}$ Zentrum für Molekulare

Neurobiologie,

Universitätsklinikum Hamburg-

Eppendorf, Hamburg, Germany

${ }^{6}$ Department of Pathology and

Immunology, Washington

University School of Medicine,

St Louis, Missouri, USA

${ }^{7}$ Institute of Immunology,

Hannover Medical School,

Hannover, Germany
\end{abstract}

\section{Correspondence to}

Dr Jérôme Avouac,

Rheumatology A Department,

Paris Descartes University,

Cochin Hospital, 27 rue du

Faubourg Saint Jacques,

Paris 75014, France;

jerome.avouac@cch.aphp.fr

$\mathrm{JA}$ and ME contributed equally.

Accepted 20 October 2012

Published Online First

17 November 2012

\section{ABSTRACT}

Objective To investigate the contribution of the adhesion receptor DNAX accessory molecule-1 (DNAM-1) in the development of dermal fibrosis on gene inactivation and targeted molecular strategies. Methods Human skin expression of DNAM-1 was determined by immunohistochemistry. Mice deficient for DNAM-1 (dnam $1^{-/-}$) and wild-type controls (dnam $1^{+/+}$) were injected with bleomycin or $\mathrm{NaCl}$. Infiltrating leucocytes, T cells, B cells and monocytes were quantified and inflammatory cytokines were measured in lesional skin of dnam $1^{-/-}$and dnam $1^{+/+}$mice. The anti-fibrotic potential of a DNAM-1 neutralising monoclonal antibody (mAb) was evaluated in the mouse model of bleomycin-induced dermal fibrosis.

Results Overexpression of DNAM-1 was detected in the skin of patients with SSc (systemic sclerosis). Dnam1 $1^{-/}$mice were protected from bleomycin-induced dermal fibrosis with reduction of dermal thickening $(75 \pm 5 \%, p=0.03)$, hydroxyproline content $(46 \pm 8 \%$, $p=0.04)$ and myofibroblast counts $(39 \pm 5 \%, p=0.01)$. Moreover, the number of $\mathrm{T}$ cells was significantly decreased in lesional skin of dnam1 $1^{-/-}$mice $169 \pm 15 \%$, $\mathrm{p}=0.0007)$. Dnam $1^{-/-}$mice also displayed decreased levels of TNF- $\alpha$ and IL-6 in lesional skin. Consistent with the gene inactivation strategy, treatment of mice with DNAM-1 neutralising mAb prevented dermal fibrosis induced by bleomycin with reduction of dermal thickness $(64 \pm 6 \%, p=0.002)$, hydroxyproline content $(61 \pm 8 \%$, $p=0.004)$ and myofibroblast counts $(83 \pm 12 \%, p=0.002)$. Conclusions An inactivation gene strategy showed that DNAM-1 exerts profibrotic effects by controlling T cell activation and cytokine release. A molecular targeted strategy confirmed that DNAM-1 neutralising mAb has potent antifibrotic properties, supporting the hypothesis that inhibition of DNAM-1 might be a promising new approach for the treatment of SSc and potentially other related fibrotic diseases.

Systemic sclerosis (SSc) is an orphan connective tissue disease of unknown aetiology with widespread microvascular damage and progressive fibrosis of the skin and internal organs. ${ }^{1}$ Early stages of SSc are characterised by vascular changes, including endothelial cell apoptosis, and perivascular inflammatory infiltrates. ${ }^{2}$ Later stages of SSc are characterised by an excessive accumulation of extracellular matrix components.

The largest group of susceptibility genes for SSc, identified by genetic association studies of single nucleotide polymorphism (SNP) markers, is related to autoimmunity and inflammatory response. ${ }^{3}$ The majority of these genes have also been recognised as risk factors for other connective tissue disorders and autoimmune diseases. ${ }^{4}$ Thus, it is crucial to determine whether these genes contribute to the expression of the fibrotic phenotype in SSc or rather reflect a shared common genetic basis of autoimmune diseases. On this line, we recently provided for the first time, translational evidence that confirms the role of one of the identified genes, STAT4, in the development of inflammation driven fibrosis, which is a hallmark of early stages of SSc. ${ }^{5}$ These stages are characterised by the infiltration of inflammatory cells in lesional tissue. Previous data showed that $\mathrm{T}$ cells represent the major component of infiltrating leucocytes, which release inflammatory and pro-fibrotic cytokines that stimulate collagen synthesis by resident fibroblasts. ${ }^{6}$ Moreover, activated $T$ cells have been suggested to play a key role in the induction of the hyperactive and altered functional phenotype of SSc fibroblasts. The DNAX accessory molecule 1 (DNAM-1) has been shown to be an important regulator of the adhesion and co-stimulation of $T$ cells and has been identified as a genetic risk factor for SSc. ${ }^{8}{ }^{9}$ DNAM-1 is a $67-\mathrm{kDa}$ type I membrane protein belonging to the immunoglobulin supergene family of receptors, containing two Ig-like domains in the extracellular region and is constitutively expressed on the majority of CD4 and CD8 T cells, monocytes, natural killer (NK) cells, platelets and a subset of B cells. ${ }^{8}$

Thus, considering the potential role of $\mathrm{T}$ cells in early stages of SSc, we hypothesised that DNAM-1 could contribute to the development of the fibrotic phenotype of SSc. To validate this hypothesis, we combined a gene activation strategy using mice lacking DNAM-1 (dnam $1^{-/}$) and a targeted molecular approach with neutralising antiDNAM-1 monoclonal antibody (mAb), to characterise the role of DNAM-1 in mice by studying bleomycin-induced dermal fibrosis, a widely used model of SSc. 


\section{MATERIALS AND METHODS}

Human skin biopsies

Paraffin embedded sections of lesional skin biopsies were obtained from 12 SSc patients and 8 healthy age- and sexmatched healthy volunteers. The median age of SSc patients was 46 years (range 25-68 years) and their median disease duration was 6 years (range 1-17 years); five had the limited cutaneous subset and seven the diffuse subset. No patients were treated with immunosuppressive or other potentially disease modifying drugs. All patients and controls signed a consent form approved by the local institutional review boards.

Bleomycin-induced dermal fibrosis in DNAM-1 deficient mice Dnam $1^{-/-}$mice have been described elsewhere. ${ }^{10}$ Wild-type C57BL/6 mice $\left(\right.$ dnam $^{+/+}$) were purchased from Janvier (Le Genest-Saint-Isle, France). Skin fibrosis was induced in 6-week-old male and female mice by local injections of bleomycin for 3 weeks; $100 \mu \mathrm{l}$ of bleomycin dissolved in $0.9 \%$ sodium chloride $(\mathrm{NaCl})$ at a concentration of $0.5 \mathrm{mg} / \mathrm{ml}$ was administered every other day by subcutaneous injections in defined areas of $1 \mathrm{~cm}^{2}$ at the upper back. Subcutaneous injections of $100 \mu \mathrm{l} 0.9 \% \mathrm{NaCl}$ were used as controls. Four different groups, consisting of two groups with dnam $1^{-/-}$mice and dnam $1^{+/+}$were analysed. One group of $\mathrm{dnam} 1^{-/-}$mice and one group of dnam $1^{+/+}$mice was challenged with bleomycin, whereas the remaining two groups were injected with $\mathrm{NaCl}$. The four groups consisted of 28 mice in total.

\section{Prevention of bleomycin-induced fibrosis with anti-DNAM-1 mAb}

To investigate whether prophylactic treatment with an anti-DNAM-1 neutralising $\mathrm{mAb}$ might protect against the development of bleomycin-induced skin fibrosis, one group of C57BL/6 mice (all males, 6-week-old) was subjected to bleomycin injections for 3 weeks and treated intraperitoneally with a neutralising $\mathrm{mAb}$ against mouse DNAM-1, at a concentration of $1.6 \mathrm{mg} / \mathrm{ml}$, initially at a dose of $400 \mu \mathrm{g}$ on day 1 , then $200 \mu \mathrm{g}$ every 5 days for 3 weeks, as in previous experiments. ${ }^{11}$ Two control groups were used: one group of mice were treated for 3 weeks with control IgG and injected with bleomycin; the other group were treated for 3 weeks with control IgG and injected with $\mathrm{NaCl}$. The three groups consisted of 18 mice in total. The local ethical committee approved all animal experiments.

\section{Evaluation of dermal thickness}

Lesional skin areas were excised, fixed in 4\% formalin and embedded in paraffin. Sections (5 $\mu \mathrm{m}$ thick) were stained with $\mathrm{H} \& \mathrm{E}$. The dermal thickness was analysed at 100-fold magnification by measuring the distance between the epidermaldermal junction and the dermal-subcutaneous fat junction at four sites from lesional skin of each mouse. ${ }^{5}$ Two independent examiners blinded to the treatment status performed the evaluation ( $\mathrm{JA}$ and ME).

\section{Assessment of the number of infiltrating leucocytes in bleomycin-treated mice}

Infiltrating leucocytes in lesional skin of $\mathrm{dnam} 1^{-/-}$and dnam $1^{+/+}$mice were quantified on $\mathrm{H} \& \mathrm{E}$ stained sections. Eight different high power fields from different tissue sites from each mouse were evaluated for mononuclear/inflammatory cells at 400-fold magnification by two independent examiners blinded to the treatment (JA and ME).
All images were captured with a Nikon Eclipse 80i microscope (Nikon, Badhoevedorp, Netherlands) equipped with a digital signal processor (DSP) 3CCD camera (Sony, Tokyo, Japan). ${ }^{5}$

\section{Collagen measurements}

The collagen content in lesional skin samples was explored by hydroxyproline assay. After digestion of punch biopsies $\left(\varnothing 3 \mathrm{~mm}\right.$ ) in $6 \mathrm{M} \mathrm{HCl}$ for three hours at $120^{\circ} \mathrm{C}$, the $\mathrm{pH}$ of the samples was adjusted to 7 with $6 \mathrm{M} \mathrm{NaOH}$. Samples were then mixed with $0.06 \mathrm{M}$ chloramine $\mathrm{T}$ and incubated for $20 \mathrm{~min}$ at room temperature. Next, 3.15 M perchloric acid and $20 \%$ p-dimethylaminobenzaldehyde were added and samples were incubated for additional $20 \mathrm{~min}$ at $60^{\circ} \mathrm{C}$. The absorbance was determined at $557 \mathrm{~nm}$ with a Spectra MAX 190 microplate spectrophotometer (Molecular Devices, Sunnyvale, California, USA).

For direct visualisation of collagen fibres, trichrome staining was performed using the Masson Trichrome Staining Kit (Sigma-Aldrich).

\section{Immunohistochemistry for $\alpha$-smooth muscle actin,} DNAM-1, CD3, CD4, CD8, CD22 and CD68

For immunohistochemistry, skin sections were deparaffinised, followed by antigen retrieval with Tris/EDTA/Tween, incubation with $5 \%$ bovine serum albumin in phosphate buffered saline for $1 \mathrm{~h}$ to block non-specific binding, and incubation with $3 \% \quad \mathrm{H}_{2} \mathrm{O}_{2}$ for $10 \mathrm{~min}$ to block endogenous peroxidase activity. Irrelevant isotype matched antibodies were used as controls in all experiments. Staining was visualised with aminoethylcarbazole, using a peroxidase substrate kit (Vector, Burlingame, California, USA).

Myofibroblasts were identified by staining for $\alpha$-smooth muscle actin ( $\alpha$-SMA), as previously described. ${ }^{5} 12$ Cells positive for $\alpha$-SMA in mouse skin sections were detected by incubation with monoclonal anti- $\alpha$-SMA antibody (clone 1A4; Sigma-Aldrich, Saint-Quentin Fallavier, France) at a dilution of $1: 1000$ for $3 \mathrm{~h}$ at room temperature. Polyclonal rabbit antimouse labelled with horseradish peroxidase (HRP) were used as secondary antibodies for $1 \mathrm{~h}$ at room temperature. The number of myofibroblasts was determined at 200-fold magnification in four different sections from each mouse by two blinded examiners (JA and ME) (an example is provided in additional figure 1).

The expression of DNAM-1 and the number of T cells in SSc patients and controls were detected by staining overnight at $4^{\circ} \mathrm{C}$ with polyclonal rabbit anti-human DNAM-1 antibody (Sigma-Aldrich) and polyclonal rabbit anti-human antibodies for CD3 (Abcam, Cambridge, UK), respectively. Polyclonal goat anti-rabbit antibodies (Dako, Glostrup, Denmark) labelled with HRP were used as secondary antibodies for $1 \mathrm{~h}$ at room temperature. The intensity of DNAM-1 immunostaining was quantified with the imageJ software (http://rsbweb.nih.gov/ij/ docs/examples/stained-sections/index.html).

To quantify the numbers of infiltrating $T$ cells, $B$ cells and monocytes, lesional skin sections from dnam $1^{-/-}$and dnam $1^{+/+}$mice were stained for CD3, CD22 and CD68, respectively. CD4 and CD8 $\mathrm{T}$ cell subsets were also quantified after staining for CD4 and CD8. Skin sections were incubated with polyclonal rabbit anti-human antibodies for $\mathrm{CD} 3$ or CD22 (Abcam), monoclonal rabbit anti-human antibodies against CD8 (Novus Biologicals, Littleton, Colorado, USA) or monoclonal mouse anti-human antibodies against CD4 or CD68 (Abcam and Novus Biologicals, respectively). Polyclonal HRP labelled goat anti-rabbit or rabbit anti-mouse immunoglobulins (Dako) were used as secondary antibodies. T cells, 
CD4 and CD8 T cell subsets, B cells and monocytes were counted in eight different sections of lesional skin of each mouse at 400-fold magnification. Counting was performed in a blinded manner by two examiners ( $J A$ and $M E$ ).

Inflammatory cytokine measurement in lesional skin samples of bleomycin-treated mice

Cytokine levels were measured in the skin of $24 \mathrm{dnam} 1^{-/-}$and dnam $1^{+/+}$mice subjected to bleomycin or $\mathrm{NaCl}$ injections (six per group). Mouse skin tissue lysate was prepared by homogenisation in modified RIPA buffer $(50 \mathrm{mM}$ Tris/ $\mathrm{HCl} \mathrm{pH} 7.5,1 \%$ Triton X-100, 0.5\% sodium deoxycholate, $0.1 \%$ sodium dodecyl sulphate, $150 \mathrm{mM} \mathrm{NaCl}$ and complete EDTA-free protease inhibitor cocktail (Roche, Mannheim, Germany)) with Precellys 24 tissue homogeniser/grinder (Ozyme, Montigny-leBretonneux, France). Tissue and cell debris was removed by centrifugation. Protein concentration was determined with the amidoblack method. ${ }^{13}$ Serum and skin lysates were assayed for the following cytokines by multiplex bead array technology (BD biosciences, Le Pont de Claix, France): tumour necrosis factor- $\alpha$ (TNF- $\alpha$ ), interleukin (IL)-6, interferon- $\gamma$ (IFN- $\gamma$ ), IL-4 and IL-10.

\section{Statistics}

Data were expressed as dot blots with mean. The MannWhitney $U$ test for non-related samples was used for statistical

\section{control}
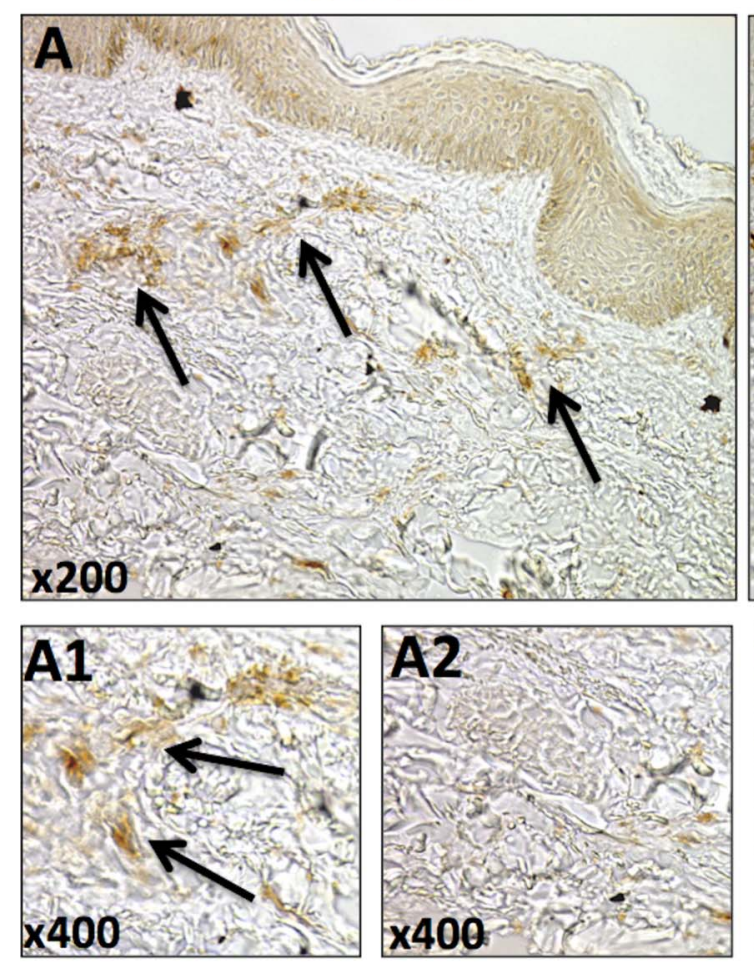

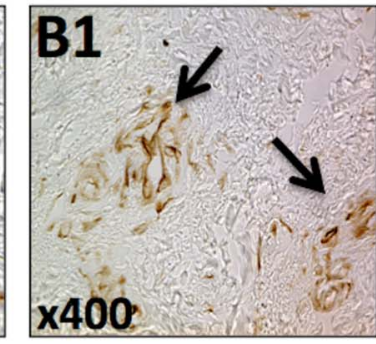

SSc
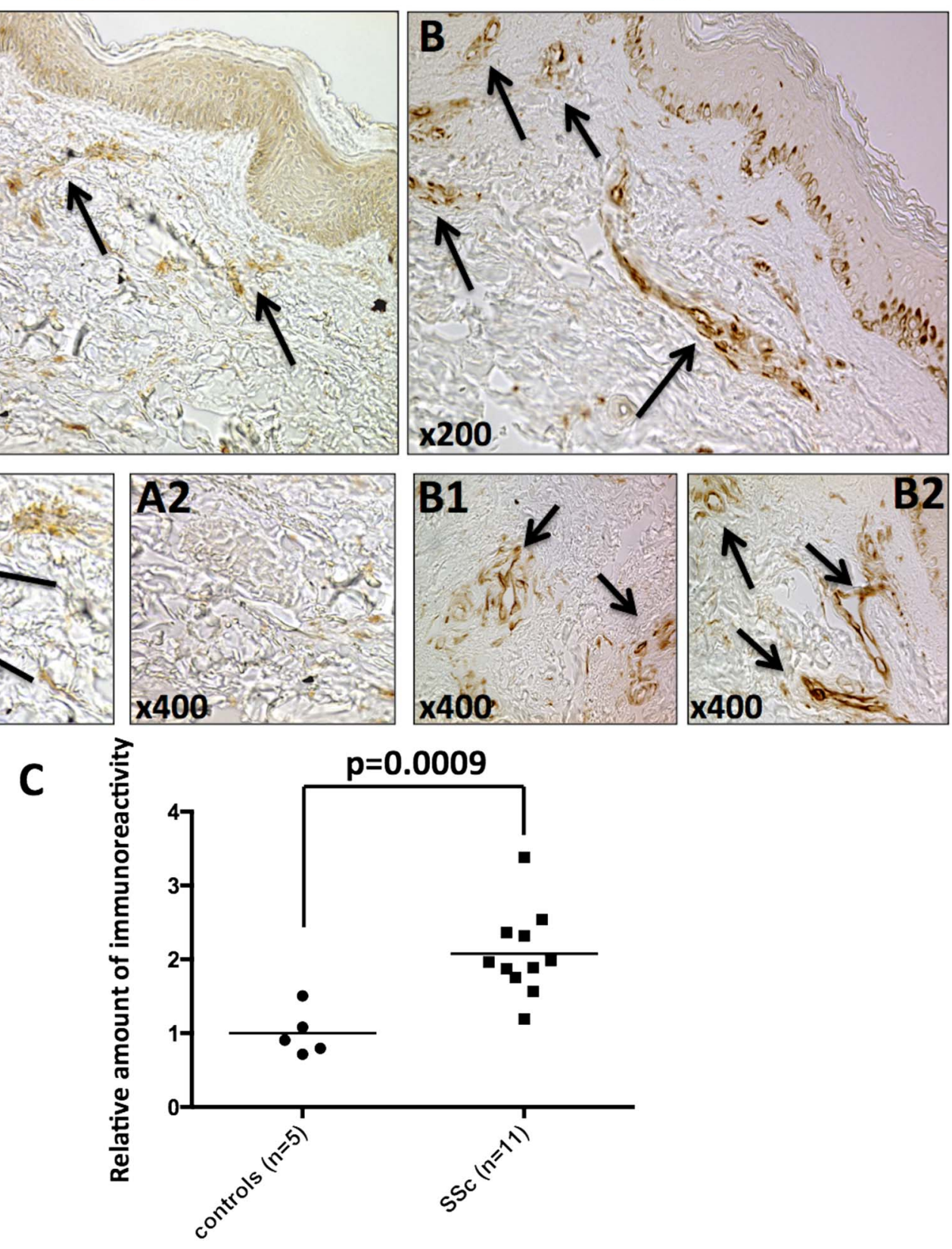

Figure 1 Increased expression of DNAM-1 in the lesional skin of systemic sclerosis (SSc) patients. (A, B) DNAM-1 protein was detected ex vivo by immunohistochemistry in SSc patients (B) compared to controls (A). Positive staining for DNAM-1 was observed in the germinal layer of the epidermis and in several cell types of the dermis (B1 and B2). (C) In addition, the intensity of immunostaining assessed by the Image J software was significantly increased in SSc patients compared to controls. The intensity of immunostaining was performed in the 11 SSc patients and 5 controls with detectable staining. Values are mean \pm SEM; arrows indicate positive DNAM-1 cells. This figure is only reproduced in colour in the online version. 

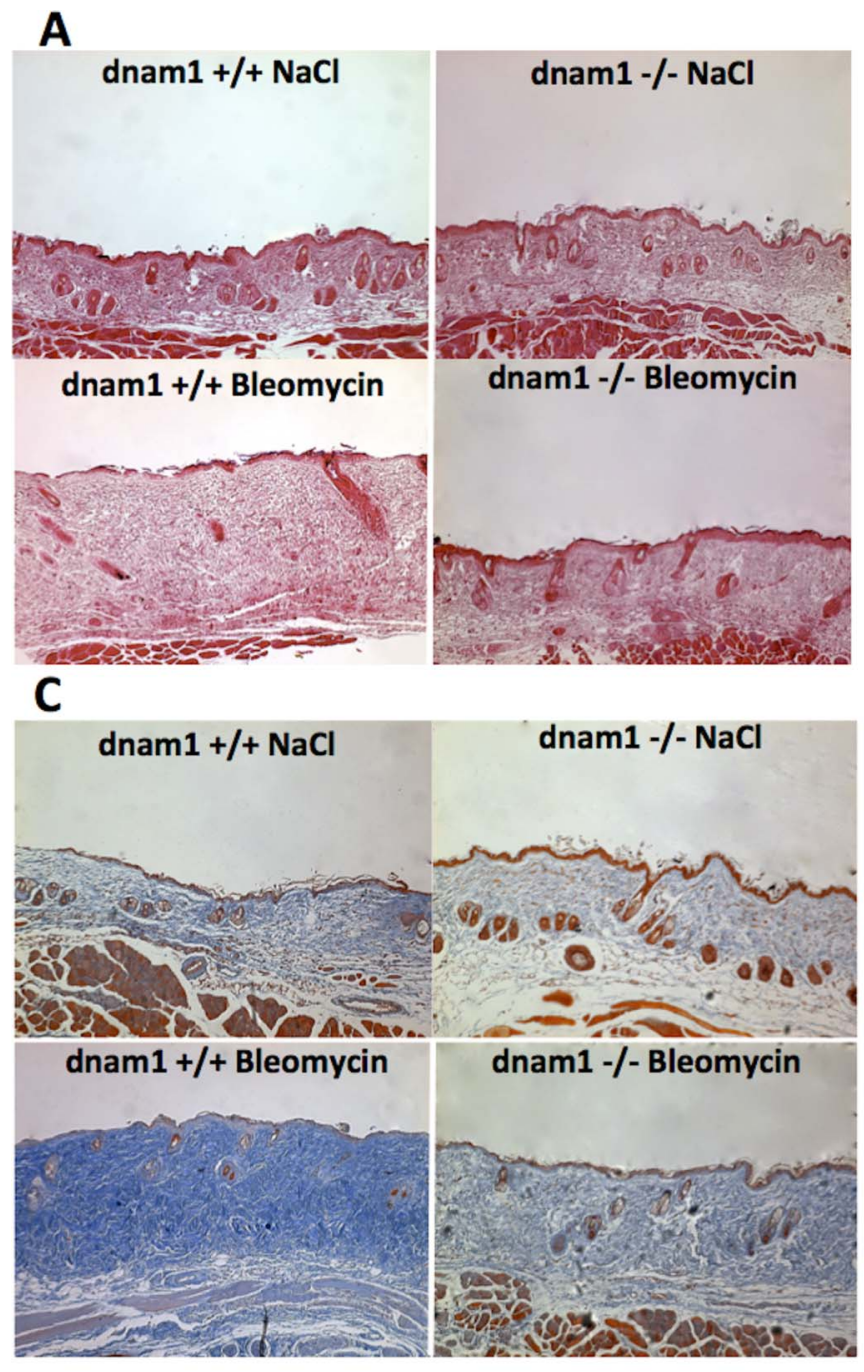

B
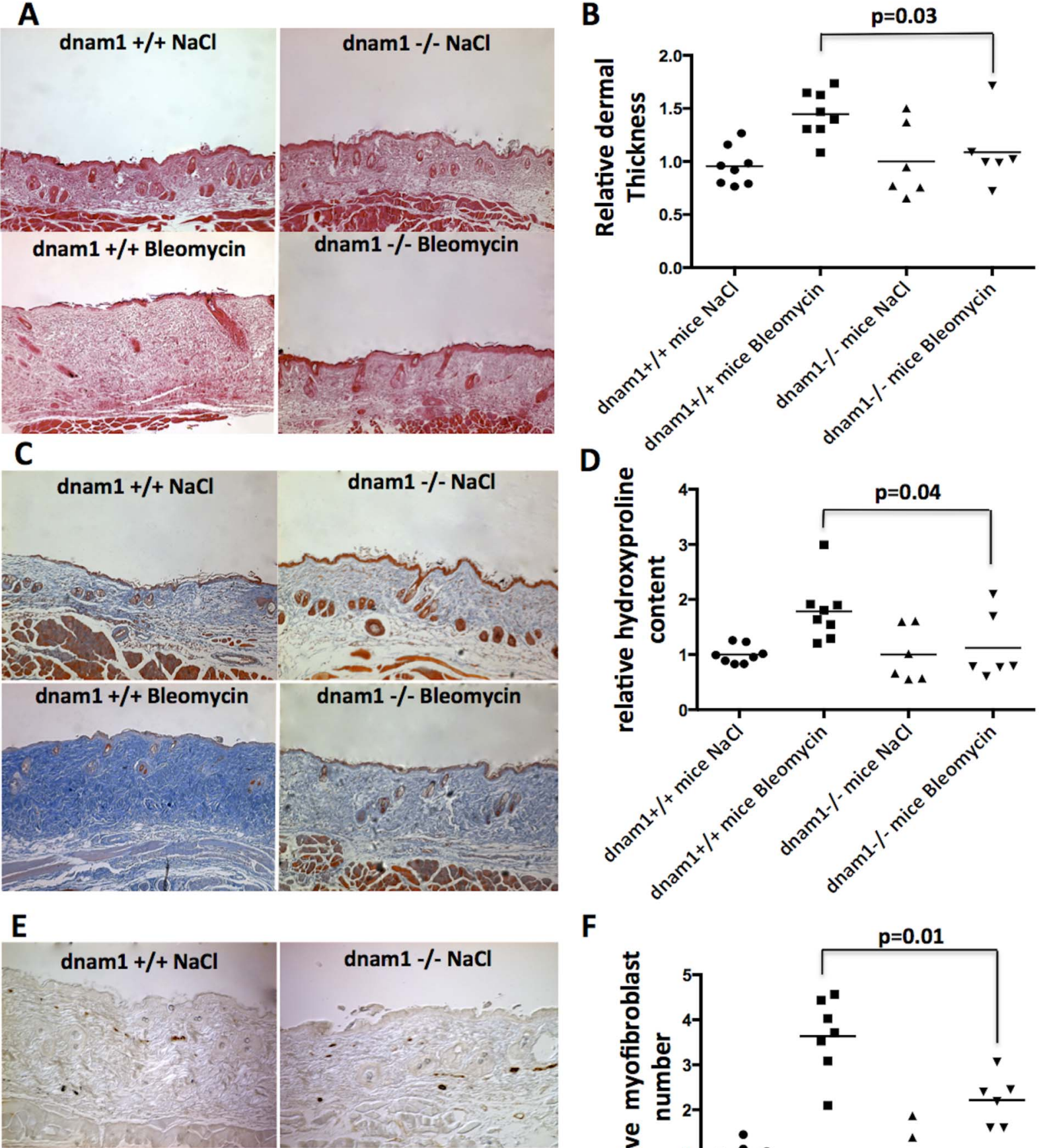

$\mathbf{F}$

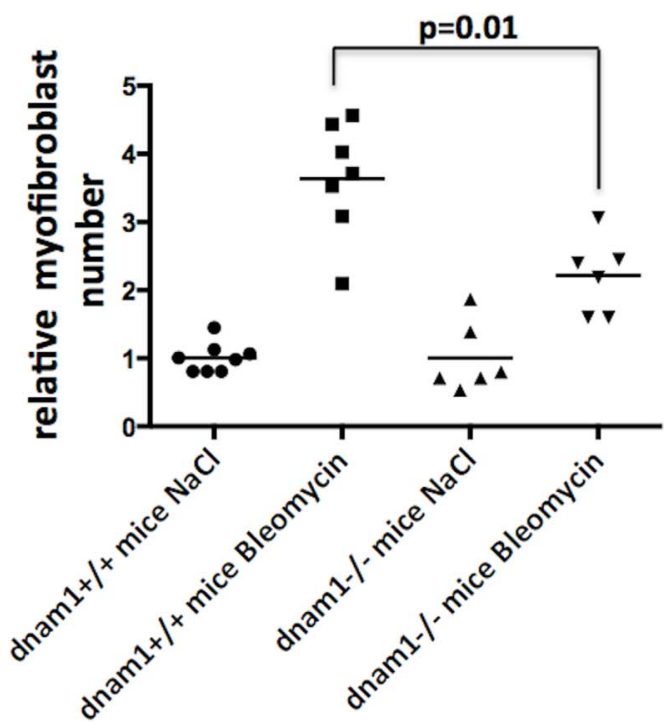

Figure 2 Dnam $1^{-/-}$mice are protected from dermal fibrosis induced by bleomycin. (A) Reduced dermal fibrosis in dnam $1^{-/-}$mice on challenge with bleomycin. Representative sections stained by H\&E at 100 -fold magnification. (B) Decreased dermal thickening in dnam $1^{-/-}$mice compared to their wild-type dnam $1^{+/+}$littermates. (C) Reduced accumulation of collagen in $\mathrm{dnam} 1^{-/-}$mice on challenge with bleomycin. Representative sections stained by trichrome staining at 100-fold magnification. (D) Reduced hydroxyproline content in lesional skin of $\mathrm{dnam}^{-{ }^{--}}$mice. (E) Reduced number of $\alpha$-smooth muscle actin positive cells by immunohistochemistry in $\mathrm{dnam}^{-/-}$mice. Representative sections at 200-fold magnification. (F) Lower myofibroblast counts in dnam $1^{-1-}$ mice; 28 mice were used for these experiments (16 wild-type mice injected with bleomycin or NaCl, and 12 dnam $1^{-/-}$mice injected with bleomycin or $\mathrm{NaCl}$ ). Values are represented by dot blots with means; dnam ${ }^{+/+}$control mice were injected with $\mathrm{NaCl}$ and defined as 1.0; the other results are normalised to this value. This figure is only reproduced in colour in the online version. 
analysis. A p value of less than 0.05 was considered statistically significant.

\section{RESULTS}

Increased expression of DNAM-1 in SSc patients

We first analysed the expression of DNAM-1 patients with SSc in comparison with controls. The expression of DNAM-1 protein was detectable in 11 of 12 SSc patients ex vivo by immunohistochemistry, but in only 5 of 8 controls (figure 1A,B). Positive staining for DNAM-1 was observed in the germinal layer of the epidermis and in several cell lines in the dermis, including perivascular inflammatory cells, such as T cells (figure 1B1,B2 and additional figure 2). In addition, the intensity of immunostaining was more abundant in SSc patients compared to controls $(p=0.0009)$ (figure $1 \mathrm{C})$. We did not find any difference for DNAM-1 expression according to age, disease duration or cutaneous subset of SSc patients.

Mice deficient for DNAM-1 are protected from bleomycin-induced dermal fibrosis

To evaluate the role of DNAM-1 in fibrosis, dnam $1^{-/-}$mice and control dnam $1^{+/+}$mice were challenged with bleomycin.
Skin architecture and dermal thickness did not differ between dnam $1^{-/-}$mice and dnam $1^{+/+}$mice injected with $\mathrm{NaCl}$, suggesting that the skin phenotype is not altered in dnam $1^{-/-}$mice compared to dnam $1^{+/+}$animals under non-fibrotic conditions (figure 2A). Following bleomycin injections, the mean \pm SEM increase in dermal thickness was $40 \pm 6 \%$ in dnam $1^{+/+}$mice, as compared with $13 \pm 8 \%$ in dnam $1^{-/-}$mice. Thus, dermal thickening on bleomycin challenge was reduced by $75 \pm 5 \%$ in dnam $1^{-1-}$ mice compared to dnam $1^{+/+}$mice $(p=0.03)$ (figure $2 \mathrm{~A}, \mathrm{~B}$ ). Consistent with decreased dermal thickening, reduced accumulation of collagen on bleomycin challenge was observed on trichrome stained skin sections of dnam $1^{-/-}$mice (figure $2 \mathrm{C}$ ). In addition, the hydroxyproline content in lesional skin of dnam $1^{-/-}$ mice was significantly lower than in the skin of dnam $1^{+/+}$mice, with a decrease of $46 \pm 8 \%(p=0.04)$ (figure $2 D$ ).

The number of myofibroblasts on challenge with bleomycin was also significantly reduced by $39 \pm 5 \%(p=0.01)$ in dnam $1^{-/-}$ mice as compared to dnam $1^{+/+}$mice (figure $2 \mathrm{E}, \mathrm{F}$ ).

DNAM-1 regulates T cell infiltration into lesional skin Inflammatory infiltrates are characteristic features of early stages of SSc that are mimicked in the mouse model of bleomycin-induced fibrosis. Infiltrating leucocytes contain
A
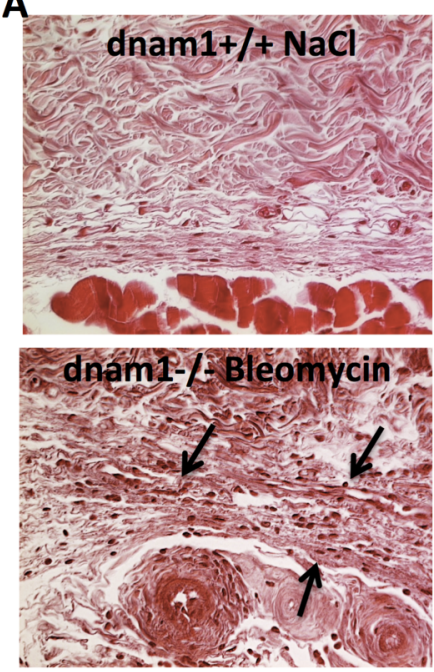

B

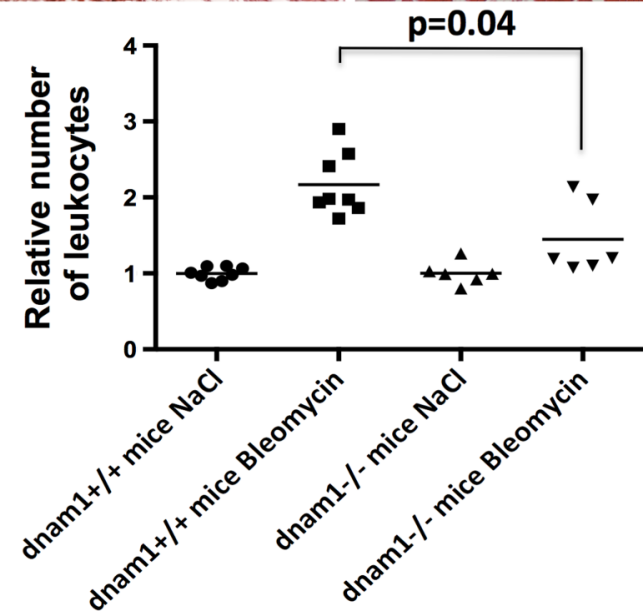

C

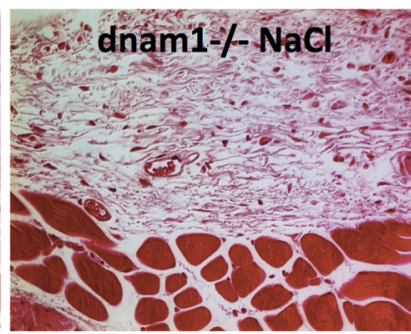

dnam1-1-Bleomycin

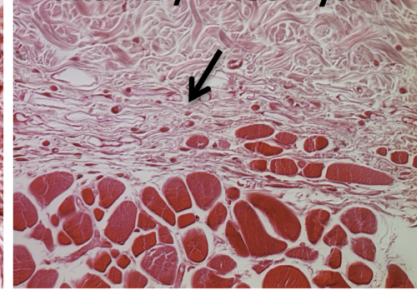

D
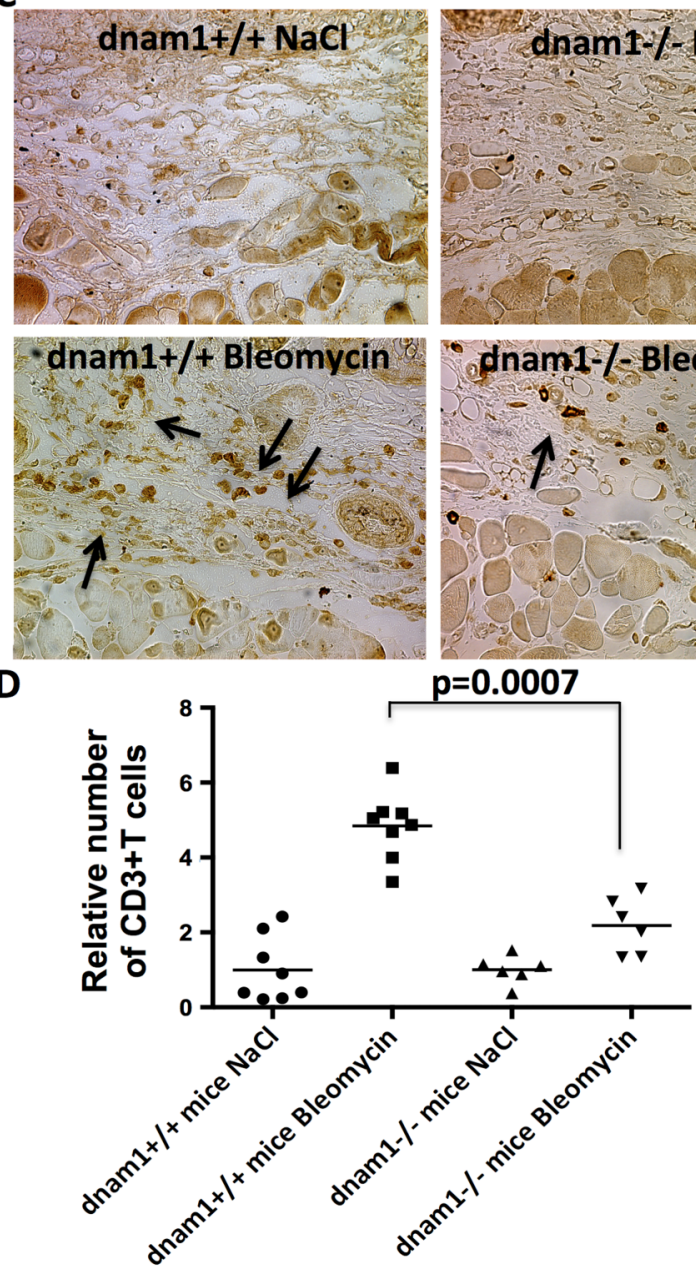

Figure 3 DNAM-1 regulates leucocyte infiltration into lesional skin. (A) Reduced inflammation in dnam $1^{-1-}$ mice challenged with bleomycin. Representative sections stained by H\&E at 400 -fold magnification. (B) Decreased leucocyte counts in lesional skin of dnam1 ${ }^{-1-}$ mice. (C) Reduced number of CD3 T cells by immunohistochemistry in lesional skin of dnam1 deficient mice challenged with bleomycin. (D) Lower CD3 T cells in dnam $1^{-/-}$mice; 28 mice were used for these experiments (16 wild-type mice injected with bleomycin or NaCl, and 12 dnam $1^{-/-}$mice injected with bleomycin or $\mathrm{NaCl}$ ). Values are represented by dot blots with means; all results are normalised to dnam $1^{+/+}$mice injected with $\mathrm{NaCl}$. Arrows indicate leucocyte infiltration (A) or positive CD3 T cells (C). This figure is only reproduced in colour in the online version. 
mostly T cells, with a perivascular distribution, and stimulate fibroblast activation and collagen synthesis by release of profibrotic factors. ${ }^{2} 14$ To analyse whether DNAM-1 influences the outcome of bleomycin-induced fibrosis by regulating leucocyte infiltration or proliferation, we next quantified the number of leucocytes in lesional skin. Inflammatory infiltrates on bleomycin treatment were significantly reduced in dnam $1^{-/-}$mice compared to dnam $1^{+/+}$mice with a reduction of $61 \pm 19 \%$ $(p=0.04)$ (figure $3 \mathrm{~A}, \mathrm{~B})$.

To investigate which leucocyte populations were affected, we first quantified the number of $\mathrm{T}$ cells in fibrotic skin. $\mathrm{T}$ cell counts were significantly lower in dnam $1^{-/-}$mice compared to dnam $1^{+/+}$mice treated with bleomycin, with a decrease of $69 \pm 15 \%(p=0.0007)$ (figure $3 C, D)$. Both CD4 and CD8 positive $\mathrm{T}$ cell numbers were reduced in dnam $1^{-/-}$mice by $71 \pm 29 \%(p=0.008)$, and $63 \pm 22 \%(p=0.01)$, respectively, compared to dnam $1^{+/+}$mice (figure $4 \mathrm{~A}, \mathrm{~B}$ ). In contrast to $\mathrm{T}$ cells, the number of $B$ cells and monocytes did not significantly differ on bleomycin challenge between $d n a m 1^{-/-}$and dnam $1^{+/+}$mice (figure 4C,D).

Reduced levels of proinflammatory cytokines in the serum and skin lysates of DNAM-1 deficient mice subjected to bleomycin injections

To further characterise how DNAM-1 regulates leucocyte infiltration and secondary fibroblast activation in the lesional skin, the levels of different cytokines were determined in the lesional skin (figure 5). Compared with dnam $1^{+/+}$mice subjected to bleomycin treatment, dnam $1^{-/-}$mice showed reduced levels of selective cytokines that have been implicated into inflammation and fibrosis such as IL-6 (reduction of $59 \pm 12 \%, \mathrm{p}=0.009$ ) and TNF- $\alpha$ (reduction of $60 \pm 15 \%, p=0.008$ ) in the lesional skin. No differences in the levels of IL-4, IL-10 and IFN- $\gamma$ were observed between dnam $1^{-/-}$and $d n a m 1^{+/+}$mice following bleomycin injections. These data suggest that DNAM-1 is associated with a proinflammatory profile, promoting tissue infiltration of $\mathrm{T}$ cells and the release of selective proinflammatory cytokines.

Anti-DNAM-1 mAb protects from the development of bleomycin-induced fibrosis

The mouse model of bleomycin-induced dermal fibrosis was used to evaluate the anti-fibrotic potential of DNAM-1 inhibition in vivo using a neutralising anti-DNAM-1 mAb. In mice injected with bleomycin and treated with control IgG, strong accumulation of thickened collagen bundles was observed (figure 6A,B). Treatment with anti-DNAM-1 mAb significantly reduced dermal thickening by $64 \pm 6 \%(p=0.002)$ (figure $6 A, B)$. These results are similar to those observed in DNAM-1 deficient mice. Consistent with reduced dermal thickening, the hydroxyproline content and the number of myofibroblasts in lesional skin were also efficiently reduced on inhibition of
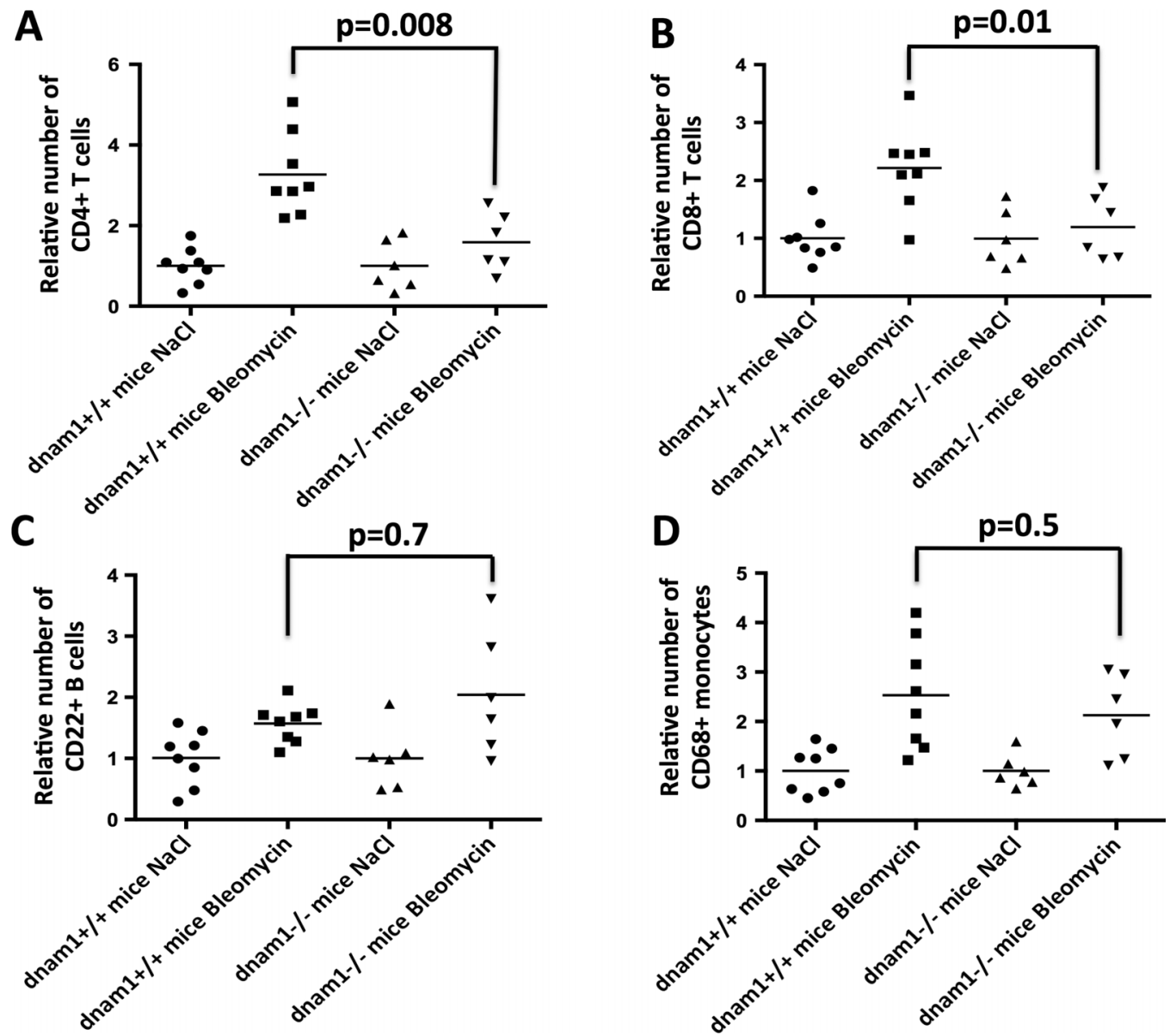

Figure 4 DNAM-1 regulates CD4 and CD8 T cell infiltration into lesional skin. (A, B) CD4 and CD8 T cell subsets were reduced in bleomycin-injected skin of dnam $1^{-/-}$mice, compared with dnam $1^{+/+}$mice. (C, D) The number of infiltrating $B$ cells and monocytes did not significantly differ on bleomycin challenge between dnam $1^{-/-}$and dnam $1^{+/+}$mice; 28 mice were used for these experiments (16 wild-type mice injected with bleomycin or $\mathrm{NaCl}$, and $12 \mathrm{dnam}^{-/-}$mice injected with bleomycin or $\mathrm{NaCl}$ ). Values are represented by dot blots with means. 

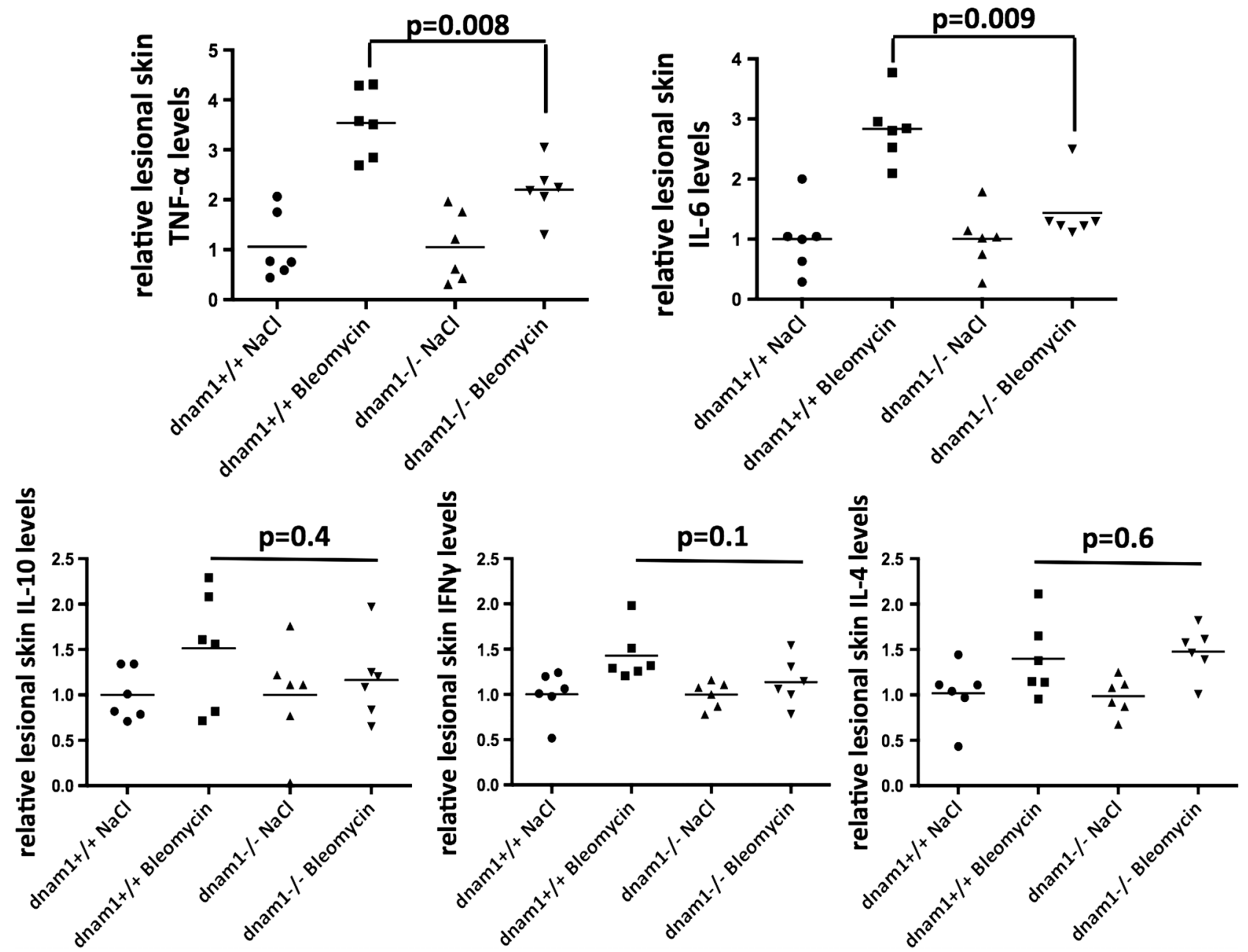

Figure 5 Reduced levels of proinflammatory cytokines in lesional skin lysates of DNAM-1 deficient mice subjected to bleomycin injections. Reduced skin levels of the inflammatory cytokines TNF- $\alpha$ and IL- 6 in dnam $1^{-/-}$mice compared with dnam $1^{+/+}$mice subjected to bleomycin. No differences in levels of IFN- $\gamma$, IL- 4 and IL-10 were observed between dnam $1^{-1-}$ or dnam $1^{+/+}$mice; 24 mice were used for these experiments (six mice per group). Values are represented by dot blots with means. All cytokine concentrations are normalised on total protein concentration. All results are normalised to dnam $1^{+/+}$mice injected with $\mathrm{NaCl}$.

DNAM-1. The hydroxyproline content decreased by $61 \pm 8 \%$ $(p=0.004)$ (figure 6C,D) and the number of myofibroblasts was reduced by $83 \pm 12 \%(p=0.002)$ (figure $6 \mathrm{E}, \mathrm{F})$.

DNAM-1 mAb was administered without serious adverse events for 3 weeks. No substantial changes were observed among mobility, activity, texture of the fur and skin integrity between mice treated with anti-DNAM-1 mAb or control IgG. Moreover, weight loss was $<10 \%$ and no significant reduction of food consumption was observed.

\section{DISCUSSION}

We demonstrate in the present study that the adhesion factor DNAM-1, a costimulatory molecule required for full activation of $\mathrm{T}$ cells, is overexpressed in lesional skin tissue of patients with SSc and contributes to the development of inflammationdriven dermal fibrosis. A gene inactivation strategy indicates that DNAM-1 exerts profibrotic effects in the model of bleomycin-induced skin fibrosis, a widely used animal model reflecting early inflammatory stages of SSc by promoting the infiltration of $T$ cells into lesional skin and regulating the cytokine balance towards a proinflammatory and profibrotic profile. In the early stages of SSc, the infiltrating leucocytes, and especially $\mathrm{T}$ cells, release inflammatory and profibrotic cytokines that activate fibroblast and stimulate collagen synthesis. ${ }^{7} 15$ As in human SSc, T cells are present in increased numbers and in an activated state in lesional skin of bleomycin challenged mice. ${ }^{6}$

CD226, encoding for the DNAM-1 protein has been identified as a risk factor of SSc and also other autoimmune diseases. $^{9} 1617$ The functional impact of the SNP rs763361 on RNA levels and/or splicing remains unclear so far. In addition, other associated SNPs in close linkage disequilibrium have been reported but the unambiguous identification of the causal gene variant is still missing. Although our results in mice suggest a functional role of DNAM-1 in fibrosis, additional investigations with a different approach will be necessary to determine the functional impact of CD226 SNP markers in SSc patients.

Our results demonstrate that DNAM-1 deficiency significantly reduced the number of infiltrating $T$ cells, in bleomycintreated mice. Although DNAM-1 is involved in the adhesion and costimulation of NK cells, we did not quantify NK cells present in lesional skin biopsies, since only minor levels of DNAM-1 expression are detected on some subsets of NK cells, with a heterogeneous profile. ${ }^{11} 18$ A reduction in numbers of infiltrating $\mathrm{T}$ cells genetically deficient for DNAM-1 was observed in a mouse model of acute graft-versus-host disease 

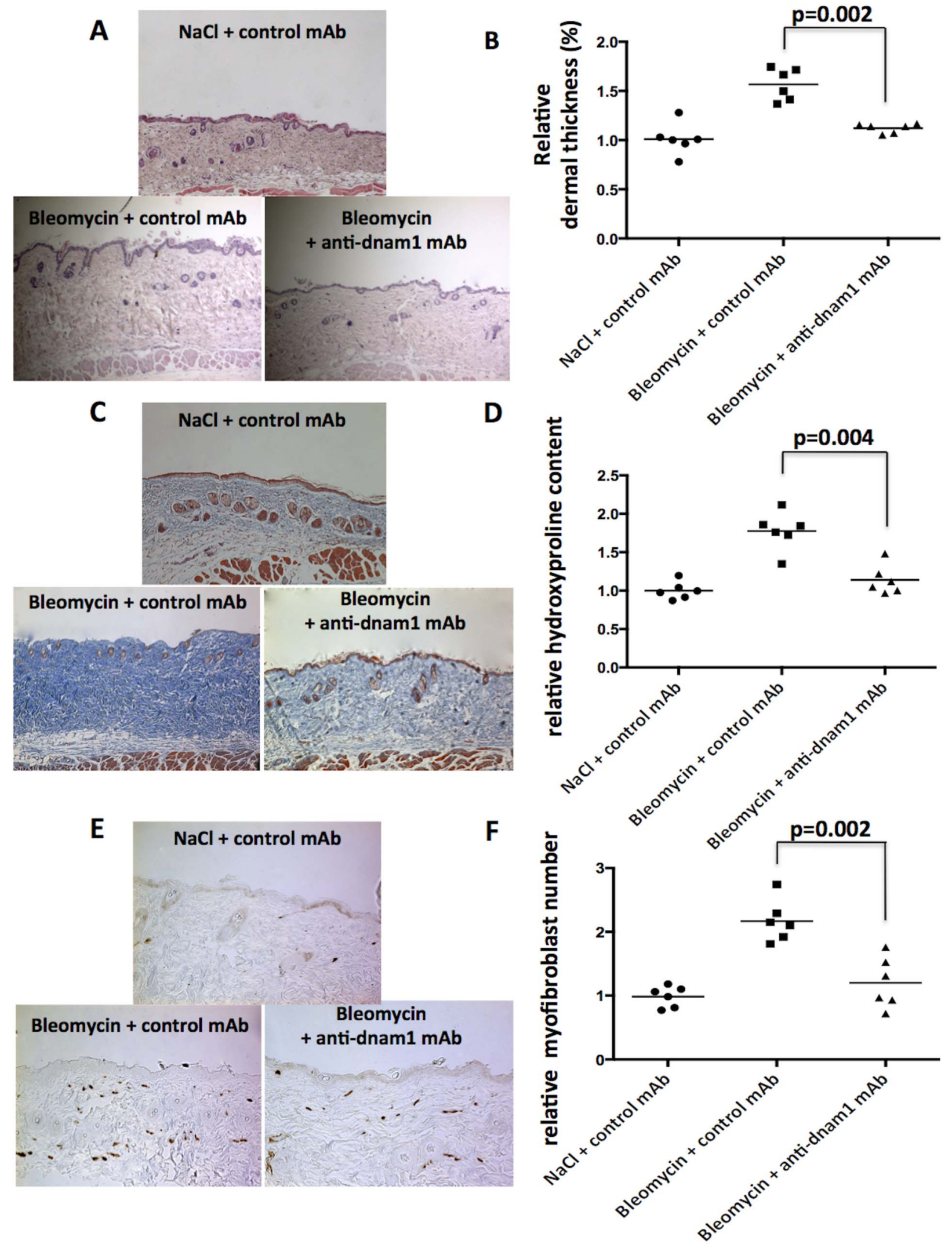

Figure 6 Bleomycin-induced skin fibrosis is prevented on DNAM-1 inhibition with a neutralising mAb. (A) In mice injected with bleomycin, a massive accumulation of thickened collagen bundles was observed. Representative sections stained by H\&E at 100-fold magnification. (B) Treatment with DNAM-1 neutralising mAb significantly reduced dermal thickening. (C, D) Consistent with reduced dermal thickening, we observed reduced accumulation of collagen on trichrome staining at 100-fold magnification (C) and decreased hydroxyproline content (D) were observed on DNAM-1 inhibition. $(E, F)$ In addition, the number of myofibroblasts was significantly reduced in mice treated by DNAM-1 neutralising mAb. Representative sections at 200-fold magnification; 18 mice were used for these experiments (six mice per group). Values are represented by dot blots with means. This figure is only reproduced in colour in the online version. 
(GVHD), a major complication of allogeneic bone marrow transplantation. ${ }^{19}$ GVHD, an immune-mediated disease that results from a complex interaction between donor and recipient immune cells, is characterised by activation and trafficking of $T$ cells to target tissues where inflammation and tissue destruction occurs. ${ }^{20}$ In this mouse model, sublethally irradiated B6C3F1 mice receiving splenocytes from DNAM-1-deficient mice lived significantly longer than mice receiving wild-type splenocytes. ${ }^{19}$ In addition, histopathological examinations showed a reduced infiltration of inflammatory cells, and especially $\mathrm{T}$ cells, in the liver and small intestine of recipients of DNAM-1 deficient splenocytes. The role of DNAM-1 has also been highlighted in experimental autoimmune encephalomyelitis (EAE). Treatment of EAE by recombinant $T$ cell receptor ligand (RTL)-551 can reverse clinical and histological signs of EAE by reducing DNAM-1+ CD4 T cells in the periphery. ${ }^{21}$ Taken together, these findings support that the blocking of DNAM-1 is associated with decreased infiltration of effector $T$ cells that mediate clinical signs of different inflammatory and immune conditions, such as inflammation-driven fibrosis, a mouse model of acute GVHD and EAE. Our findings may thus reflect a general effect of DNAM-1 on inflammation rather that an effect linked to a specific SSc genetic susceptibility, and DNAM-1 may have an impact mainly at the initiating inflammatory phase of the disease.

We demonstrate that DNAM-1 promotes the release of IL-6 and TNF- $\alpha$ from infiltrating leucocytes. Significantly reduced levels of these cytokines were found in DNAM-1 deficient mice treated with bleomycin in comparison to wild-type mice. These cytokines have been implicated in the activation of fibroblasts, collagen synthesis and subsequent fibrosis. IL- 6 is overexpressed by endothelium and fibroblasts in the lesional skin of SSc patients, as well as in bleomycin-induced skin fibrosis and tight skin mice. ${ }^{22}{ }^{24}$ Dermal fibroblasts from SSc patients were reported to constitutively produce up to 30-fold higher levels of IL-6 than those of healthy controls. ${ }^{25}$ IL- 6 promotes fibrosis by enhancing inflammation and is a potent inducer of excessive collagen production and proliferation of SSc fibroblasts, notably by exerting an autocrine regulation of fibroblasts. ${ }^{26}$ Thus, increased levels of Il-6 promoted by DNAM-1 may redirect the focus on other cell types involved in fibrosis and targeted by these cytokines, in which DNAM-1 expression has not been assessed. Blocking of the IL-6 receptor using MR16-1, an anti-IL-6 receptor monoclonal antibody, has been shown to alleviate dermal thickness, collagen production and myofibroblast infiltration in the mouse model of bleomycin-induced skin fibrosis. $^{27}$

Expression of TNF- $\alpha$ is found during early stages of SSc. ${ }^{28}$ Preclinical studies indicate that inhibition of TNF- $\alpha$ might exert antifibrotic effects in early inflammatory stages of SSc. Inhibition of TNF- $\alpha$ in bleomycin-induced dermal fibrosis resulted in a significant reduction of dermal thickness, collagen accumulation and the number of infiltrating myofibroblasts. ${ }^{29}$ Similar results were also obtained for pulmonary fibrosis. ${ }^{30} 31$ However, these promising results were not confirmed in clinical studies.

Levels of IL-10, IL-4 and IFN- $\gamma$ were not significantly different between dnam $1^{-/-}$and dnam $1^{+/+}$mice subjected to bleomycin injections. However, it is noteworthy that IL-4 and IFN- $\gamma$ levels did not significantly increase on bleomycin injections in wild-type mice, which differs from previous published reports. 56

To confirm the results obtained with DNAM-1 deficient mice, we performed a complementary molecular targeting strategy using a neutralising $\mathrm{mAb}$ specifically recognising murine DNAM-1. Using this antibody, DNAM-1 expression was shown on murine thymocytes and mature peripheral $T$ cells. On injection into mice, this antibody caused a partial depletion of CD8 T cells. ${ }^{11}$ In addition, anti-DNAM-1 mAb has previously been shown to suppress the development of acute GVHD by suppressing donor CD8 $\mathrm{T}$ cell proliferation in recipient mice. ${ }^{19}$ We observed a significant decrease of dermal thickness and collagen production in mice treated with this $\mathrm{mAb}$, similar to the reduction obtained in DNAM-1 deficient mice. Thus, the role of DNAM-1 in dermal inflammation-driven fibrosis is supported by two independent and complementary strategies of DNAM-1 inhibition. Further studies with additional mouse models of established fibrosis or inflammationindependent fibrosis would be useful to validate the promising findings obtained in the bleomycin model.

One limitation of our study was related to the quantification of myofibroblasts, defined as $\alpha$-SMA positive cells. Since $\alpha$-SMA also stains microvessels, the distinction between myofibroblasts (spindle-shaped cells) and pericytes was challenging in some cases.

We did not observe any signs of toxicity of the anti-DNAM-1 $\mathrm{mAb}$ during the study period, supporting the good safety profile of this antibody in the preclinical setting in mice.

In summary, we demonstrate with two complementary approaches that inhibition of DNAM-1, a critical new target in autoimmune disorders, significantly ameliorates dermal inflammation-driven fibrosis. DNAM-1 displays profibrotic effects by promoting the infiltration of leucocytes, especially $\mathrm{T}$ cells, into lesional skin and by stimulating the release of cytokines involved in both inflammatory and fibrotic processes. In addition, a molecular targeting strategy using a DNAM-1 neutralising $\mathrm{mAb}$ confirmed the potent antifibrotic properties of DNAM-1 inhibition. Our findings might have direct translational implications, and inhibition of DNAM-1 might be a promising new approach for the treatment of SSc and potentially other fibrotic diseases.

Acknowledgements Plateformes d'Immuno-biologie (M. Andrieu), De Morphologie/ Histologie (M. Favier) et d'imagerie cellulaire (P. Bourdoncle) de l'institut Cochin, Paris, France and Prof. Catherine Chaussain, EA2496, Université Paris Descartes, Faculté de Chirurgie Dentaire, Montrouge, France

Contributors JA, YA: study conception. JA, ME, MT, BR, MP: data acquisition. JA, ME, MF, MC, GB, GC, JHWD, YA: data analysis. JA, ME, MT, MF, GC, GB, AK, GC, JHWD, YA: manuscript preparation.

Funding INSERM, Société Française de Rhumatologie, Association des Sclérodermiques de France, Fondation pour la recherche Médicale, Arthritis Foundation, CMH Research Projects No. 00000023728. Additional grant support was provided by grant A40 of the Interdisciplinary Center of Clinical Research (IZKF) in Erlangen and grants DI 1537/1-1, DI 1537/2-1, DI 1537/4-1, AK 144/1-1 and SCHE 1583/7-1 from the Deutsche Forschungsgesellschaft. This project was supported by research grant from SERVIER research group

\section{Competing interests None.}

Provenance and peer review Not commissioned; externally peer reviewed. Correction notice This article has been corrected since it was published Online First. Figure 6 has been corrected.

\section{REFERENCES}

1. Allanore $\mathbf{Y}$, Avouac $\mathrm{J}$, Wipff $\mathrm{J}$, et al. New therapeutic strategies in the management of systemic sclerosis. Expert Opin Pharmacother 2007;8:607-15.

2. Gabrielli A, Avvedimento EV, Krieg T. Scleroderma. N Engl J Med 2009;360:1989-2003.

3. Allanore $\mathbf{Y}$, Dieude P, Boileau C. Genetic background of systemic sclerosis: autoimmune genes take centre stage. Rheumatology (Oxford) 2010;49:203-10.

4. Allanore $\mathbf{Y}$, Dieude P, Boileau C. Updating the genetics of systemic sclerosis. Curr Opin Rheumatol 2010;22:665-70. 
5. Avouac J, Furnrohr BG, Tomcik M, et al. Inactivation of the transcription factor STAT-4 prevents inflammation-driven fibrosis in animal models of systemic sclerosis. Arthritis Rheum 2011;63:800-9.

6. Yamamoto T. The bleomycin-induced scleroderma model: what have we learned for scleroderma pathogenesis? Arch Dermatol Res 2006;297:333-44.

7. Gu YS, Kong J, Cheema GS, et al. The immunobiology of systemic sclerosis. Semin Arthritis Rheum 2008;38:132-60.

8. Shibuya A, Campbell D, Hannum C, et al. DNAM-1, a novel adhesion molecule involved in the cytolytic function of T lymphocytes. Immunity 1996;4:573-81.

9. Dieude P, Guedj M, Truchetet ME, et al. Association of the CD226 Ser(307) variant with systemic sclerosis: evidence of a contribution of costimulation pathways in systemic sclerosis pathogenesis. Arthritis Rheum 2011;63:1097-105.

10. Gilfillan S, Chan CJ, Cella M, et al. DNAM-1 promotes activation of cytotoxic lymphocytes by nonprofessional antigen-presenting cells and tumors. J Exp Med 2008;205:2965-73

11. Seth S, Georgoudaki AM, Chambers BJ, et al. Heterogeneous expression of the adhesion receptor CD226 on murine NK and T cells and its function in NK-mediated killing of immature dendritic cells. J Leukoc Biol 2009;86:91-101.

12. Avouac J, Palumbo K, Tomcik M, et al. Inhibition of AP-1 signaling abrogates TGF-ss mediated activation of fibroblasts and prevents experimental fibrosis. Arthritis Rheum 2011;64:1642-52.

13. Kamel H, Brown DH, Ottaway JM, et al. Comparison of gold levels and distribution in guinea pig serum. Arthritis Rheum 1978;21:441-6.

14. Prescott RJ, Freemont AJ, Jones CJ, et al. Sequential dermal microvascular and perivascular changes in the development of scleroderma. J Pathol 1992;166:255-63.

15. Yamamoto T. Scleroderma-pathophysiology. Eur J Dermatol 2009;19:14-24

16. Maiti AK, Kim-Howard X, Viswanathan P, et al. Non-synonymous variant (Gly307Ser) in CD226 is associated with susceptibility to multiple autoimmune diseases. Rheumatology (Oxford) 2010;49:1239-44.

17. Hafler JP, Maier LM, Cooper JD, et al. CD226 Gly307Ser association with multiple autoimmune diseases. Genes Immun 2009;10:5-10.

18. Dardalhon V, Schubart AS, Reddy J, et al. CD226 is specifically expressed on the surface of Th1 cells and regulates their expansion and effector functions. J Immunol 2005; 175:1558-65.
19. Nabekura T, Shibuya K, Takenaka E, et al. Critical role of DNAX accessory molecule-1 (DNAM-1) in the development of acute graft-versus-host disease in mice. Proc Natl Acad Sci USA 2010:107:18593-8.

20. Briones J, Novelli S, Sierra J. T-cell costimulatory molecules in acute-graft-versus host disease: therapeutic implications. Bone Marrow Res 2011;2011:976793.

21. Sinha S, Miller LM, Subramanian S, et al. RTL551 treatment of EAE reduces CD226 and T-bet + CD4 T cells in periphery and prevents infiltration of T-bet + IL-17, IFN-gamma producing T cells into CNS. PLoS One 2011;6:e21868.

22. Koch AE, Kronfeld-Harrington LB, Szekanecz Z, et al. In situ expression of cytokines and cellular adhesion molecules in the skin of patients with systemic sclerosis. Their role in early and late disease. Pathobiology 1993;61:239-46.

23. Yamamoto T, Takagawa S, Katayama I, et al. Animal model of sclerotic skin. I: Local injections of bleomycin induce sclerotic skin mimicking scleroderma. J Invest Dermatol 1999:112:456-62.

24. Yamamoto T, Takagawa S, Katayama I, et al. Anti-sclerotic effect of transforming growth factor-beta antibody in a mouse model of bleomycin-induced scleroderma. Clin Immunol 1999;92:6-13.

25. Kadono T, Kikuchi $\mathrm{K}$, Ihn $\mathrm{H}$, et al. Increased production of interleukin 6 and interleukin 8 in scleroderma fibroblasts. J Rheumatol 1998;25:296-301.

26. Wipff J, Bonnet P, Ruiz B, et al. Association study of serotonin transporter gene (SLC6A4) in systemic sclerosis in European Caucasian populations. J Rheumatol 2010;37:1164-7.

27. Kitaba S, Murota $\mathrm{H}$, Terao $\mathrm{M}$, et al. Blockade of interleukin-6 receptor alleviates disease in mouse model of scleroderma. Am J Pathol 2012;180:165-76.

28. Distler JH, Schett G, Gay S, et al. The controversial role of tumor necrosis factor alpha in fibrotic diseases. Arthritis Rheum 2008;58:2228-35.

29. Koca SS, Isik A, Ozercan $\mathrm{H}$, et al. Effectiveness of etanercept in bleomycin-induced experimental scleroderma. Rheumatology (Oxford) 2008;47:172-5.

30. Piguet PF, Vesin C. Treatment by human recombinant soluble TNF receptor of pulmonary fibrosis induced by bleomycin or silica in mice. Eur Respir $J$ 1994; 7:515-8.

31. Farivar AS, Mackinnon-Patterson B, McCourtie AS, et al. Obliterative airway disease in rat tracheal allografts requires tumor necrosis factor alpha. Exp Mol Pathol 2005;78:190-7. 


\section{ARD}

Critical role of the adhesion receptor DNAX accessory molecule-1 (DNAM-1) in the development of inflammation-driven dermal fibrosis in a mouse model of systemic sclerosis

Jérôme Avouac, Muriel Elhai, Michal Tomcik, Barbara Ruiz, Manuel Friese, Melanie Piedavent, Marco Colonna, Gunter Bernhardt, André Kahan, Gilles Chiocchia, Jörg H W Distler and Yannick Allanore

Ann Rheum Dis 2013 72: 1089-1098 originally published online November 17, 2012 doi: 10.1136/annrheumdis-2012-201759

Updated information and services can be found at: http://ard.bmj.com/content/72/6/1089

These include:

Supplementary Material

References

Email alerting service
Supplementary material can be found at:

http://ard.bmj.com/content/suppl/2012/11/17/annrheumdis-2012-2017 59.DC1.html

This article cites 31 articles, 9 of which you can access for free at: http://ard.bmj.com/content/72/6/1089\#BIBL

Receive free email alerts when new articles cite this article. Sign up in the box at the top right corner of the online article.
Topic
Collections
Articles on similar topics can be found in the following collections

Connective tissue disease (4234)

Genetics (964)

Immunology (including allergy) (5117)

\section{Notes}

To request permissions go to:

http://group.bmj.com/group/rights-licensing/permissions

To order reprints go to:

http://journals.bmj.com/cgi/reprintform

To subscribe to BMJ go to:

http://group.bmj.com/subscribe/ 